\title{
A Case of Advanced Gastric Cancer with Poor Performance Status Which Improved by Chemotherapy
}

\author{
Kohei Shitara Daisuke Takahari \\ Department of Clinical Oncology, Aichi Cancer Center Hospital, Nagoya, Japan
}

\section{Key Words}

Gastric cancer · Poor performance status · Chemotherapy

\begin{abstract}
Introduction: The prognosis of advanced gastric cancer patients, especially those with poor performance status (PS), is generally dismally poor. Patients with PS 3-4 are usually ineligible for participation in clinical studies and are managed with only best supportive care.

Case Report: A 63-year-old male with advanced gastric cancer was admitted to our hospital. His PS was markedly impaired (Eastern Cooperative Oncology Group PS 4), with dyspnea secondary to lymphangitis, pleuritis and pericarditis). He also had bilateral leg paralysis due to multiple bone metastases. He was treated with chemotherapy using 5fluorouracil and leucovorin for 14 days with pericardial drainage followed by intrapericardial infusion of cisplatin. He was also treated with radiotherapy for bone metastasis. The patient required $5 \mathrm{l} / \mathrm{min}$ oxygen therapy at the start of chemotherapy, but his dyspnea was improved by day 14 and he no longer required supplemental oxygen therapy. His leg paralysis also improved with the radiation therapy. His PS was significantly improved with this multimodal treatment modality, and he was ultimately discharged with chemotherapy with oral fluoropyrimidine.
\end{abstract}

Conclusion: This case suggests that multimodal therapy including chemotherapy may be beneficial in advanced gastric cancer patients even in the setting of poor PS. Further study might be required to confirm the benefit of chemotherapy in this patient population.

\section{Introduction}

Performance status (PS) is a strong independent prognostic factor for survival in patients with advanced gastric cancer [1,2]. In general, patients selected for participation in clinical trials are required to have an Eastern Cooperative Oncology Group (ECOG) PS 
$\leq 2$. In fact, the majority of patients in cancer clinical trials have an ECOG PS 0-1, with less than 10\% having a PS 2. Few clinical trials targeting ECOG PS 3 or 4 gastrointestinal cancer patients have been reported $[3,4]$; thus, such patients are not generally considered to be candidates for chemotherapy. According to the US National Comprehensive Cancer Network (NCCN) clinical practice guidelines for gastric cancer [5], patients with an ECOG PS 3-4 should only be offered best supportive care. Despite these recommendations, some gastric cancer patients in clinical practice with a deteriorated PS wish to receive chemotherapy. Here we report a case of an advanced gastric cancer patient with poor PS (ECOG PS 4) whose PS improved with chemotherapy and radiotherapy.

\section{Case Presentation}

A 63-year-old Japanese male was admitted to our hospital with worsening dyspnea, cough and anorexia for 1 month. He also had noticed bilateral leg paralysis for 2 weeks. One year before admission, he was diagnosed as having gastric cancer with pathological evidence of a poorly-differentiated adenocarcinoma for which he refused to undergo any surgical or medical treatment. On admission, his physical examination was notable for decreased respiratory sounds in bilateral lower lungs. His oxygen saturation was $86 \%$ on room air and $96 \%$ with $21 /$ min oxygen administration. He also demonstrated bilateral leg weakness. Chest X-ray on admission showed an enlarged cardiac silhouette, with a cardiothoracic ratio of $60 \%$. His laboratory results were notable for increased serum liver enzymes (aspartate transaminase 1,149 IU/l, alanine transaminase $965 \mathrm{IU} / \mathrm{l}$, lactate dehydrogenase 1,580 IU/l). Echocardiography showed pericardial effusions with triphasic flow with increased retrograde amplitude in the inferior vena cava on duplex Doppler sonogram, which was suspected to be caused by increased right heart pressure during the cardiac cycle. Therefore, emergent pericardiocentesis was performed, and the drainage tube was left in the pericardial space. The total volume of effusions was approximately $500 \mathrm{ml}$ at day $1,300 \mathrm{ml}$ at day 2 and $200 \mathrm{ml}$ at day 3 . Malignant adenocarcinoma cells were detected in cytologic analysis of the effusion. Although his liver dysfunction was improved by pericardial drainage, his dyspnea worsened, which required increment of $\mathrm{O}_{2}$ administration (5 l). A computed tomography (CT) scan showed extensive infiltration of the lung and bilateral pleural effusions, compatible with lymphangitic carcinomatosis (fig. 1a, b). Abdominal CT scan showed para-aortic lymph node metastasis and ascites (fig. 2a, b). Bone scan revealed multiple bone metastases at cervical and thoracic vertebrae (C7 to Th7). Although he had PS 4 with dyspnea requiring oxygen administration, he strongly wished to receive chemotherapy. Therefore, combination therapy consisting of 5-fluorouracil (5-FU) and leucovorin (LV) was initiated. The dose and administration schedule were planned as follows: infusional 5-FU $\left(250 \mathrm{mg} / \mathrm{m}^{2}\right)$ in $24 \mathrm{~h}$ and $\mathrm{LV}\left(25 \mathrm{mg} / \mathrm{m}^{2}\right)$ in $1 \mathrm{~h}$, on days $1-14$ every 21 days. Although his respiratory status was transiently worsened and oxygen administration was increased to $7 \mathrm{l}$ on day 3 (fig. 3a), it improved thereafter, and oxygen administration was discontinued at day 14. Because his leg weakness worsened soon after the initiation of chemotherapy, radiotherapy for bone metastasis (total 30 Gy) was also performed. The amount of pericardial effusions from the drainage tube decreased to 100 $\mathrm{ml} /$ day at the end of 5-FU plus LV treatment, and the catheter was removed after intracavitary treatment with a $30 \mathrm{mg}$ bolus of cisplatin. His course was complicated by grade 2 stomatitis secondary to the chemotherapy; this improved within 1 week. His oral intake improved concurrent with the improvement of PS, and chemotherapy using oral fluoropyrimidine of S-1 was initiated after completion of infusional chemotherapy, approximately 2 months after his initial presentation. Chest Xray and CT scan prior to $S-1$ chemotherapy showed improved extensive lung infiltration and ascites (fig. $1 c, d, 2 c, d, 3 b$ ). His PS was well maintained (PS 1) with chemotherapy administered on an outpatient basis. However, his tumor returned after 5 months of oral chemotherapy. Although he was treated with second-line chemotherapy using paclitaxel, he passed away 2 months later, approximately 9 months after his initial admission.

\section{Discussion}

As a result of disease progression, patients with gastric cancer experience several complications, including anorexia, fatigue, abdominal distension and dyspnea, which can lead to deterioration of general patient status. The prognosis of advanced gastric cancer patients, particularly those with an ECOG PS 3-4, is dismally poor. Usually, best 
supportive care without chemotherapy is offered to these patients [5]. However, according to our previous report [6] which evaluated 116 gastrointestinal cancer patients with PS 3-4 who received chemotherapy (including 37 gastric cancer patients), 32.7\% achieved a tumor response, or a decrease in accumulated fluid, or a decline in tumor markers, resulting in a survival benefit compared to the other patients without any effect (6.9 months vs. 2.2 months, $\mathrm{p}<0.001$ ). Also, improvement in the PS for a duration of more than 4 weeks was seen in $13.8 \%$ of the patients with a low incidence of toxicityrelated death $(0.8 \%)$. These results and this case report suggest that chemotherapy might be beneficial in some gastric cancer patients, even those with a poor PS. However, the effect of chemotherapy may not be sufficient to achieve an improvement in PS, as evidenced by our use of multimodal therapy in this patient. Therefore, further study might be required to investigate which patients might gain benefit from chemotherapy. For example, gefitinib for patients with lung adenocarcinoma harboring mutation in epidermal growth factor receptor results in PS improvement of 79\% and median survival time of 17.8 months even in patients with poor PS [7]. The development of targeted therapies for gastric cancer should also allow the delivery of tailored treatment more adequate for individual patients through molecular profiling of their tumors. Finally, it is important to consider the patient's opinion due to the lack of sufficient evidence and the modest improvement in survival obtained with chemotherapy in advanced gastric cancer.

\section{Conclusion}

Our case suggests that multimodal therapy including chemotherapy may be beneficial in advanced gastric cancer patients even in the setting of poor PS.

\section{Consent}

Written informed consent was obtained from the patient's wife for publication of this case report and accompanying images. A copy of the written consent is available for review by the Editor-in-Chief of this journal. 


\begin{tabular}{c|l|l|l}
$\begin{array}{c}\text { Cose Reports in } \\
\text { Ondolady }\end{array}$ & $\begin{array}{l}\text { Case Rep Oncol 2010;3:262-267 } \\
\text { D01: 10.1159/000319169 }\end{array}$ & Published online: July 21, 2010 & $\begin{array}{l}\text { O 2010 S. Karger AG, Basel } \\
\text { ISSN 1662-6575 } \\
\text { www.karger.com/cro }\end{array}$ \\
\hline
\end{tabular}

Fig. 1. a, b CT scan prior to chemotherapy with 5-FU plus leucovorin. Extensive infiltration of the lung and bilateral pleural effusions are shown, compatible with lymphangitis carcinomatosis. c, d CT scan prior to chemotherapy with S-1. Lung infiltration and bilateral pleural effusions had nearly disappeared.

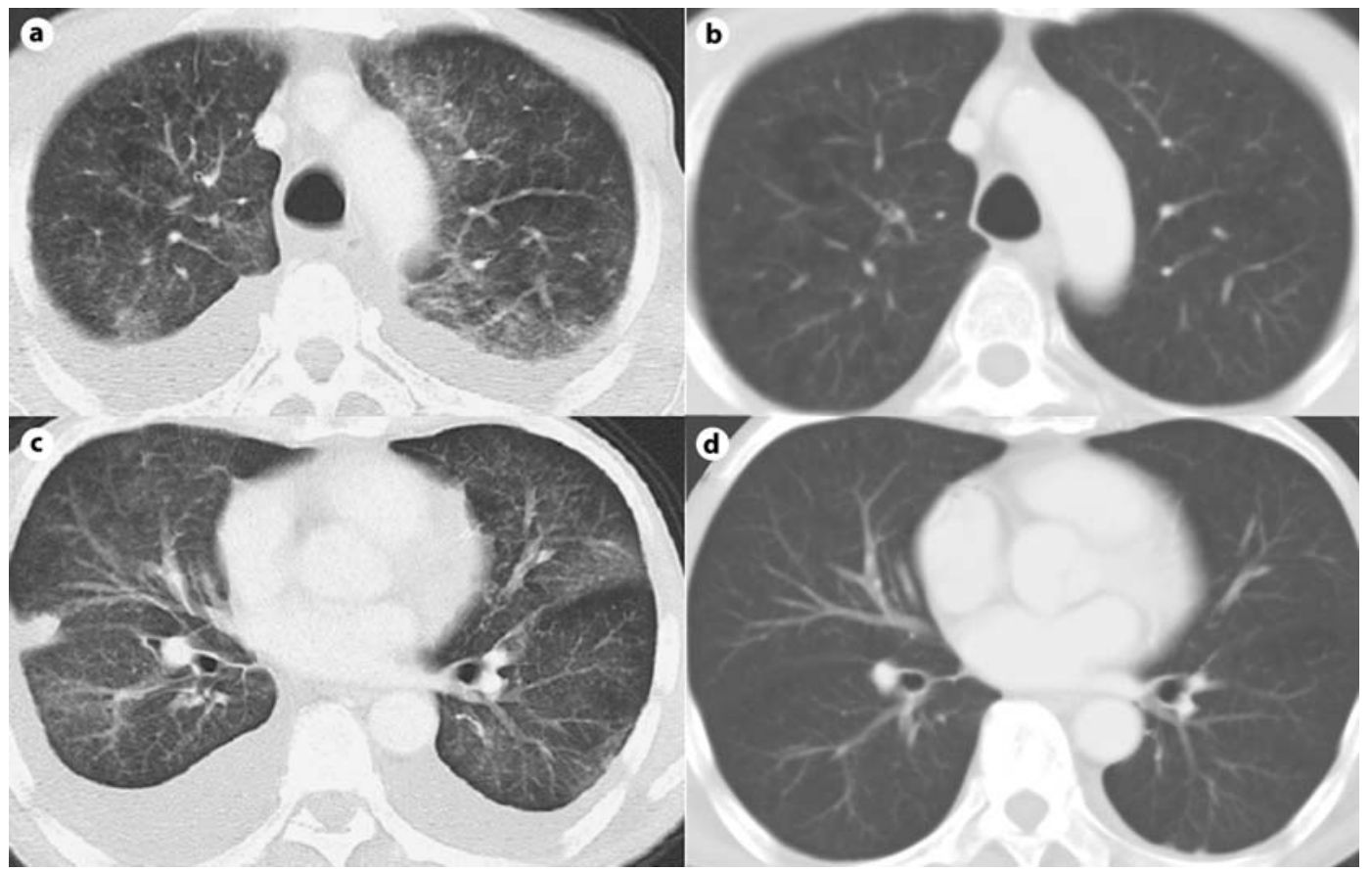

Fig. 2. a, b CT scan prior to chemotherapy with 5-FU plus leucovorin. Bilateral pleural effusions, ascites and para-aortic lymph node metastases were seen. Pericardial tube was inserted at apex (white arrowhead). c, d CT scan prior to chemotherapy with S-1. Bilateral pleural effusions and ascites had nearly disappeared. Para-aortic lymph node metastases were reduced in size.

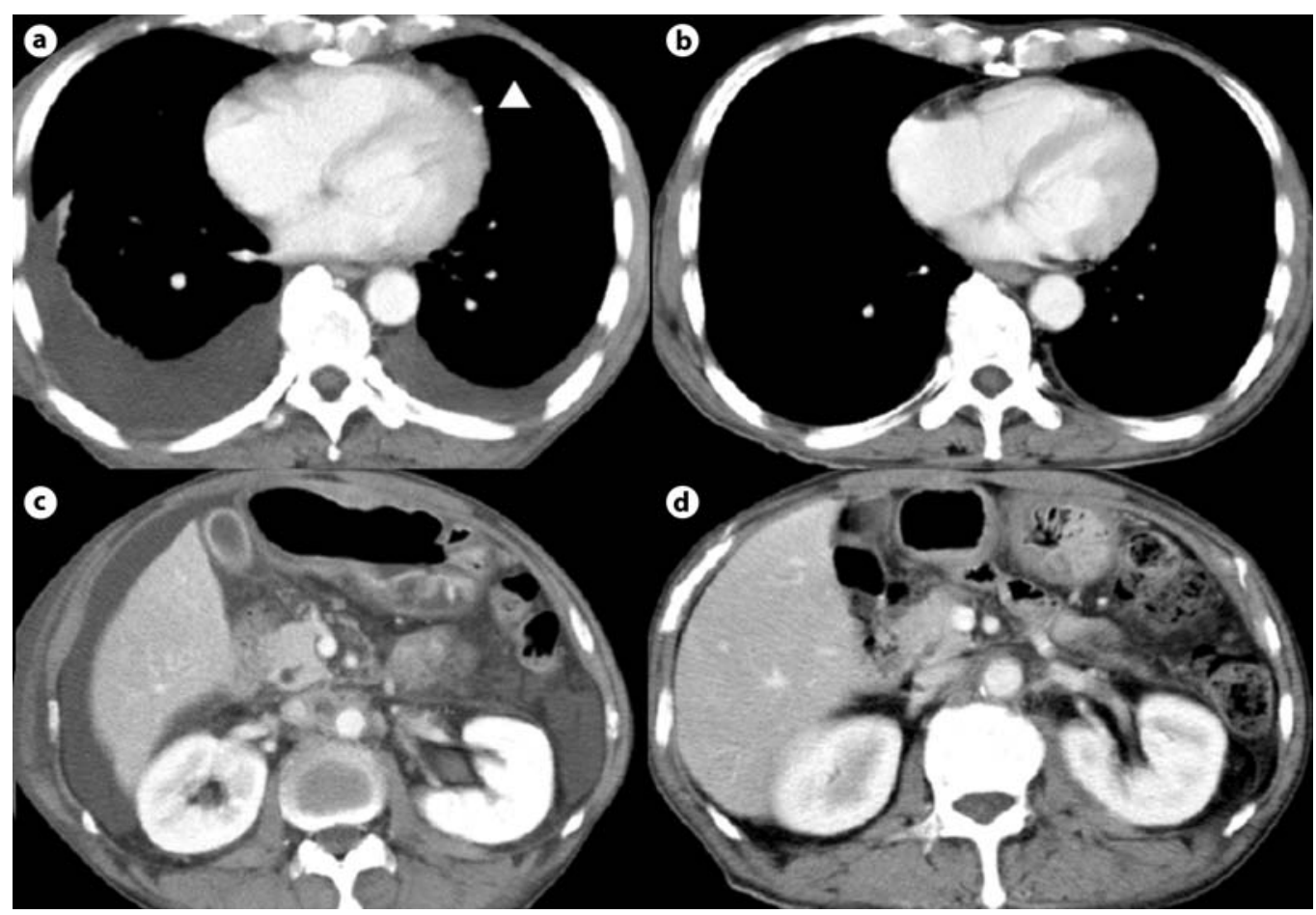




\begin{tabular}{c|l|l|l}
$\begin{array}{c}\text { Case Reports in } \\
\text { Ondolady }\end{array}$ & $\begin{array}{l}\text { Case Rep Oncol 2010;3:262-267 } \\
\text { D0I: 10.1159/000319169 }\end{array}$ & Published online: July 21, 2010 & $\begin{array}{l}\text { O 2010 S. Karger AG, Basel } \\
\text { ISSN 1662-6575 } \\
\text { www.karger.com/cro }\end{array}$ \\
\hline
\end{tabular}

Fig. 3. a Chest X-ray at day 3 of chemotherapy with 5-fluorouracil and leucovorin, demonstrating extensive infiltration of the lung. b Chest X-ray prior to chemotherapy with S-1. Extensive lung infiltration has disappeared.

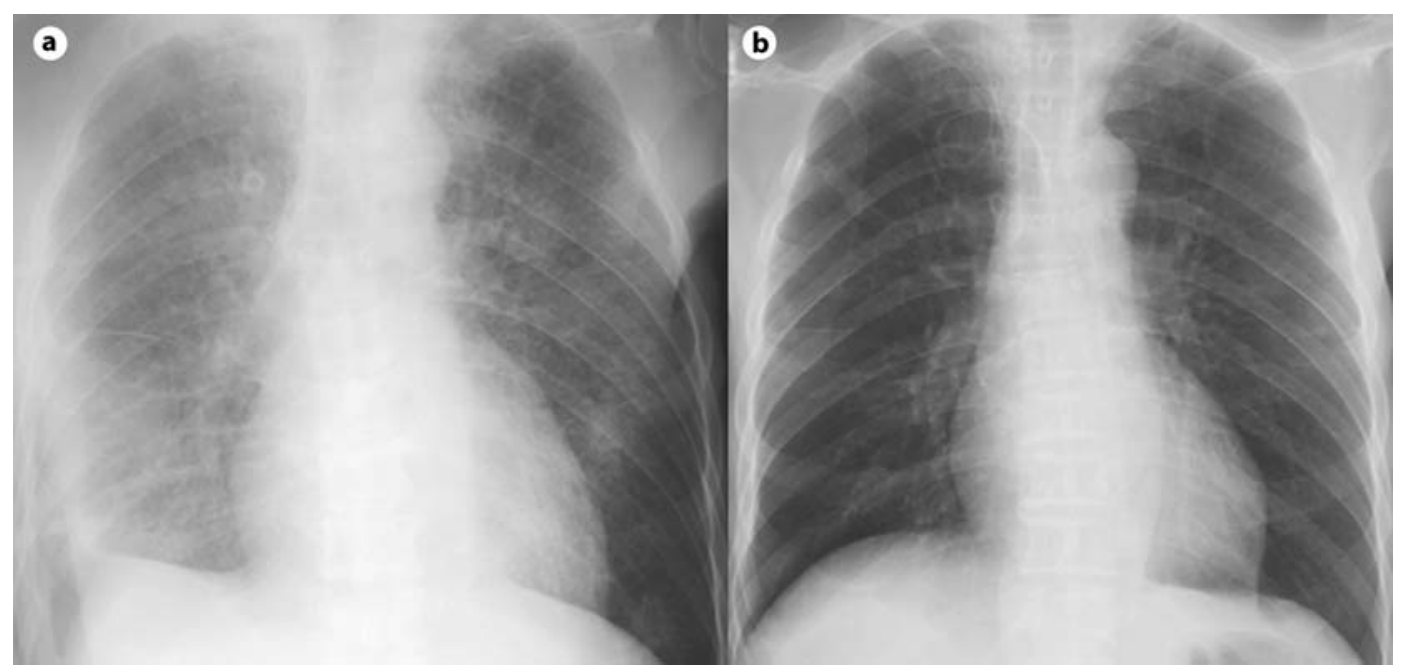




\section{References}

1 Buonadonna A, Lombardi D, De Paoli A, Bidoli E, Frustaci S: Adenocarcinoma of the stomach: univariate and multivariate analyses of factors associated with survival. Suppl Tumori 2003;2:S31-S34.

-2 Chau I, Norman AR, Cunningham D, Waters JS, Oates J, Ross PJ: Multivariate prognostic factor analysis in locally advanced and metastatic esophago-gastric cancer pooled analysis from three multicenter, randomized, controlled trials using individual patient data. J Clin Oncol 2004;22:2395-2403.

-3 Jeung HC, Rha SY, Shin SJ, Ahn JB, Noh SH, Roh JK, Chung HC: A phase II study of S-1 monotherapy administered for 2 weeks of a 3-week cycle in advanced gastric cancer patients with poor performance status. Br J Cancer 2007;97:458463.

- 4 Hsu CH, Yeh KH, Chen LT, Liu JM, Jan CM, Lin JT, Chen YC, Cheng AL: Weekly 24-h infusion of high-dose 5-fluorouracil and leucovorin in the treatment of advanced gastric cancers. An effective and low-toxic regimen for patients with poor general condition. Oncology 1997;54:275-280.

5 NCCN Clinical Practice Guidelines in Oncology: Gastric Cancer. NCCN Practice Guidelines in Oncology. Version 2.2009.

6 Shitara K, Munakata M, Kasai M, Muto O, Sakata Y: Prolongation of survival and improvement in performance status following palliative chemotherapy in gastrointestinal cancer patients with a poor performance status. Oncology 2008;74:135-142.

7 Inoue A, Kobayashi K, Usui K, Maemondo M, Okinaga S, Mikami I, Ando M, Yamazaki K, Saijo Y, Gemma A, Miyazawa H, Tanaka T, Ikebuchi K, Nukiwa T, Morita S, Hagiwara K; North East Japan Gefitinib Study Group: First-line gefitinib for patients with advanced non-small-cell lung cancer harboring epidermal growth factor receptor mutations without indication for chemotherapy. J Clin Oncol 2009;27:1394-1400. 